\title{
Effect of Propranolol Alone and in Combination with Pyrimethamine on Acute Murine Toxoplasmosis
}

\author{
Mahbobeh Montazeri ${ }^{1,2}$; Ahmad Daryani ${ }^{1,2,}$; Mohammadali Ebrahimzadeh ${ }^{3}$; Ehsan \\ Ahmadpour ${ }^{4}$; Mehdi Sharif ${ }^{1,2}$; Shahabeddin Sarvi ${ }^{1,2}$ \\ ${ }_{1}^{1}$ Toxoplasmosis Research Center, Mazandaran University of Medical Sciences, Sari, IR Iran \\ ${ }_{2}^{2}$ Department of Parasitology and Mycology, Sari Medical School, Mazandaran University of Medical Sciences, Sari, IR Iran \\ ${ }^{3}$ Pharmaceutical Sciences Research Center, School of Pharmacy, Mazandaran University of Medical Sciences Sari, IR Iran \\ 4 Infectious and Tropical Diseases Research Center, Tabriz University of Medical Sciences, Tabriz, IR Iran \\ ${ }^{*}$ Corresponding author: Ahmad Daryani, Department of Parasitology and Mycology, Sari Medical School, Mazandaran University of Medical Sciences, Sari, IR Iran. Tel: +98-1513241031, \\ Fax:+98-1513543249, E-mail: daryanii@yahoo.com
}

Received: August 6, 2014; Revised: December 3, 2014; Accepted: January 26, 2015

Background: Toxoplasmosis is a public health problem worldwide. This complication principally affects immunodeficient patients and pregnant women. Toxoplasma gondii is an opportunistic parasite, causing severe illness among and death of high-risk individuals and treatment is becoming increasingly difficult owing to side effects and low efficacies of drugs.

Objectives: In this study, we investigated the anti-Toxoplasma gondii efficacy of propranolol in vivo.

Materials and Methods: This study was performed in two separate pre-treatment and post-treatment groups. In each group, 18 female Balb/c mice in six subgroups ( $\mathrm{n}=3$ ) were used to assess the anti-Toxoplasma effect of propranolol at $2 \mathrm{and} 3 \mathrm{mg} / \mathrm{kg} /$ day, pyrimethamine at $50 \mathrm{mg} / \mathrm{kg} /$ day, propranolol at 2 and $3 \mathrm{mg} / \mathrm{kg} /$ day plus pyrimethamine, and phosphate-buffered saline(PBS; as negative control). Treatment was performed 4, 24, and 48 hours before and after an intraperitoneal challenge of $1 \times 10^{3}$ tachyzoites of the virulent RH strain of T. gondii, in pre-treatment and post-treatment groups. Mice peritoneal exudates were collected on the seventh day after the challenge and parasite numbers were recorded as percent of growth inhibition and survival rate.

Results: In the pre-treatment group, results showed that propranololat 2 and $3 \mathrm{mg} / \mathrm{kg}$ combined with pyrimethamine was more effective in inhibiting growth of tachyzoites ( $86 \%$ and $98 \%$, respectively) when compared with propranolol at 2 and $3 \mathrm{mg} / \mathrm{kg}$ ( $37 \%$ and $39 \%$, respectively) and pyrimethamine (41\%) alone. In the post-treatment group, all combined treatments significantly reduced parasite load. The growth inhibition of tachyzoites in mice receiving propranolol ( 2 and $3 \mathrm{mg} / \mathrm{kg}$ ) was $75 \%$ and 51\%, with the mean tachyzoites count being $1526 \pm 171.4$ and $2948 \pm 1452.8$, respectively, compared with pyrimethamine treatment outcome, which represents 99.9\% growth inhibition.

Conclusions: Our results demonstrated the promising prophylactic and therapeutic effects of propranolol against T. gondii infection. Propranolol also increases the efficacy of pyrimethamine in combination therapies.

Keywords: Propranolol; Pyrimethamine; Mice; Toxoplasma

\section{Background}

Toxoplasmosis is an opportunistic infection caused by the obligate intracellular protozoan parasite Toxoplasma gondii. The infection has a worldwide distribution among humans and other warm-blooded animals (1). Toxoplasma gondii has a complex life cycle with asexual and sexual portions. After the ingestion of oocysts (sporozoites) or tissue cysts (bradyzoites), the released zoites evolve into tachyzoites in the host gut lumen. Tachyzoites could penetrate the intestinal epithelium, invading cells and replicating during an acute infection. In immune-competent hosts, tachyzoites evolve into bradyzoites and form cysts in the tissues; the infection then proceeds onto the latent phase. Humans usually remain asymptomatic unless immunosuppression occurs, whereupon the organism can be encysted and reactivated $(2,3)$. Therefore, toxoplasmo- sis is considered a serious and life-threatening illness for immunocompromised patients (such as those with HIV) and newborns. All at-risk groups require anti-Toxoplasma therapy (4).

The first choice for the treatment of toxoplasmosis in immunocompromised patients or after confirmed fetal infection at 18 weeks of gestation or later is a combination of pyrimethamine and sulfadiazine (5). Spiramycin is administered during pregnancy to prevent perinatal transmission. In addition, other drugs such as clindamycin, azithromycin, and atovaquone can be used for clinical toxoplasmosis (5-7). Current chemotherapy cannot destroy tissue cysts and do not eliminate intracellular parasites, and thus is still deficient. Furthermore, these drugs often have many side effects; for instance, pyri-

Copyright (C) 2015, Ahvaz Jundishapur University of Medical Sciences. This is an open-access article distributed under the terms of the Creative Commons Attribution-NonCommercial 4.0 International License (http://creativecommons.org/licenses/by-nc/4.0/) which permits copy and redistribute the material just in noncommercial usages, provided the original work is properly cited. 
methamine inhibits dihydrofolate reductase; therefore, folinic acid must be administered in combination with pyrimethamine to protect the bone marrow from suppressive effects $(1,5,7)$.

Toxoplasma gondii enters host cells via endocytosis. The parasite cytoskeleton probably plays an important role in its motility, invasion, and endodyogeny. Some research has been carried out on drugs that affect the parasite's complex cytoskeleton. Disruption of any of these essential functions would be expected to kill or inhibit the parasite. It is known that cell membrane stabilizing drugs can change the resistance of the cell membrane, leading to interference with microfilament function and blockage of the actin gel (8-10).

The authors in the previous study have shown that ketotifen (11), colchicine, chromolyn sodium, and propranolol act as cell membrane stabilizing drugs, and thus can inhibit T. gondii penetration into nucleotide cells (unpublished data). In order to further study these possibilities, we examined propranolol's anti-Toxoplasma activity in a murine model as a continuation of our previous studies.

\section{Objectives}

Considering the need for alternative drugs with fewer toxic side effects for the treatment of toxoplasmosis, we evaluated the anti-Toxoplasma activity of propranolol in a murine model of acute toxoplasmosis.

\section{Materials and Methods}

\subsection{Animals}

Six-week old inbred female Balb/c mice weighing 18-20 g were used for this experiment. The study underwent ethical review and was approved by the ethics committee of Mazandaran university of medical sciences. The care and use of the experimental animals complied with local animal welfare laws, guidelines, and policies. All experimental mice were housed under standard laboratory conditions with an average temperature of $20-25^{\circ} \mathrm{C}$, and were given drinking water and a regular mouse diet.

\subsection{Parasites}

Tachyzoites of the virulent RH strain of T. gondii were used for experimentation. They were obtained routinely by intraperitoneal passage in Swiss-Webster female mice (12). To prepare the fresh tachyzoites, $0.5 \mathrm{~mL}$ of the parasite suspension in sterile Phosphate-Buffered Saline (PBS; $\mathrm{pH}=7.4$ ) containing $100 \mathrm{IU} / \mathrm{mL}$ penicillin and 100 $\mu \mathrm{g} / \mathrm{mL}$ streptomycin was injected into mice peritoneum; the tachyzoites were then harvested after 3 - 4 days from peritoneal exudates. The concentration of tachyzoites was determined by counting in a hemacytometer using light microscope. For the challenge, suspensions were adjusted to $1 \times 10^{3}$ tachyzoites in $1 \mathrm{~mL}$ PBS and inoculated intraperitoneally into female Balb/c mice $(13,14)$.

\subsection{Experimental Design and Groups}

The current study was performed in pre-treatment and post-treatment groups. In each study, 18 mice in six groups $(\mathrm{n}=3)$ were used to assess the anti-Toxoplasma ef fect of the following drugs: (1) propranolol $2 \mathrm{mg} / \mathrm{kg} / \mathrm{day}$, (2) propranolol $3 \mathrm{mg} / \mathrm{kg} /$ day, (3) pyrimethamine $50 \mathrm{mg} /$ $\mathrm{kg} /$ day + propranolol $2 \mathrm{mg} / \mathrm{kg} /$ day, (4) pyrimethamine $50 \mathrm{mg} / \mathrm{kg} /$ day + propranolol $3 \mathrm{mg} / \mathrm{kg} /$ day, (5) pyrimethamine $50 \mathrm{mg} / \mathrm{kg} /$ day (positive control), and (6) PBS (negative control). Propranolol and pyrimethamine were a gift from Sohahelal pharmaceutical company (Tehran, Iran). The drugs were dissolved and diluted in PBS for immediate use. Initially, to control drug side effects, a preliminary experiment was conducted on mice receiving the same dose of drugs for 3 days. No mortality or clinically significant toxicity was observed.

In the pre-treatment group, drug injections were performed 48,24 , and 4 hours before the challenge $\left(1 \times 10^{3} \mathrm{~T}\right.$. gondii tachyzoites $(\mathrm{mL})$. In another set of experiments, in the post-treatment group, drugs were injected 4,24 , and 48 hours after a challenge of the same dose. Infected mice were kept for seven days and the animals were monitored daily for mortality. Peritoneal exudates were collected on the seventh day after the challenge. To collect the fluids, $3 \mathrm{~mL}$ PBS containing an antibiotic was injected into mice peritoneum, and after mild shaking, the exudates were aspirated and the parasite load determined using hemacytometer $(12,15)$.

\subsection{Statistical Analysis}

Statistical analysis was performed using SPSS software. Differences between the test and control groups were analyzed by Mann-Whitney U test. A P-value less than 0.05 was considered statistically significant.

\section{Results}

\subsection{Anti-Toxoplasma Effect of Propranolol in Pre- Treatment Groups}

The in vivo anti-Toxoplasma effects of propranolol (2 and $3 \mathrm{mg} / \mathrm{kg} /$ day), pyrimethamine $(50 \mathrm{mg} / \mathrm{kg} /$ day $)$, propranolol combined with pyrimethamine, and PBS on the growth of tachyzoites are summarized in Table 1. These results show that propranolol at 2 and $3 \mathrm{mg} / \mathrm{kg} /$ day in combination with pyrimethamine was more effective in inhibiting tachyzoite growth than propranolol alone at 2 and $3 \mathrm{mg} / \mathrm{kg} /$ day (37\% and 39\%, respectively) and pyrimethamine alone (41\%). There was a significant difference in the inhibitory effects of $2 \mathrm{mg} / \mathrm{kg} /$ day propranolol and pyrimethamine $(\mathrm{P}<0.05)$. Furthermore, the mean Toxoplasma growth inhibition rate of propranolol combined with pyrimethamine treatment was dose-dependent, with propranolol $3 \mathrm{mg} / \mathrm{kg} /$ day plus pyrimethamine having excellent activities and producing maximum growth inhibition in the pre-treatment group $(\mathrm{P}<0.05)$. 
There was a significant difference in the growth inhibition rates between all drug-treated and untreated groups (negative control) $(\mathrm{P}<0.001)$.

\subsection{Anti-Toxoplasma Effect of Propranolol in Post- Treatment Groups}

The in vivo anti-Toxoplasma effects of 2 and $3 \mathrm{mg} / \mathrm{kg} /$ day of propranolol, $50 \mathrm{mg} / \mathrm{kg} /$ day of pyrimethamine, and a combination of the above-mentioned drugs are presented in Table 2 . The results indicate that these drugs significantly reduced parasite load, especially when used in combination. The growth inhibition of tachyzoites in mice receiving propranolol 2 and $3 \mathrm{mg} / \mathrm{kg}$ was $75 \%$ and
$51 \%$, respectively, with the mean tachyzoite count being $1526 \pm 171.4$ and $2948 \pm 1452.8$, respectively, compared with pyrimethamine treatment outcome, which represents $99.9 \%$ growth inhibition. The difference between the two doses of propranolol was dose-dependent but not significant $(\mathrm{P}>0.05)$. There was no difference in anti-Toxoplasma activity between mice receiving pyrimethamine alone and pyrimethamine combined with propranolol $3 \mathrm{mg} / \mathrm{kg}$ ( $\mathrm{P}>0.05)$. All drugs significantly reduced parasite load as compared to the negative control group $(\mathrm{P}<0.001)$.

Mice receiving propranolol $2 \mathrm{mg} / \mathrm{kg} /$ day in combination with pyrimethamine had a decreased parasite load compared to those receiving pyrimethamine $(\mathrm{P}<0.05)$.

\begin{tabular}{|c|c|c|}
\hline Drug/Dose, mg & Tachyzoites No. $\left(\times 10^{4}\right)$ & \% Growth Inhibition ${ }^{a}$ \\
\hline \multicolumn{3}{|l|}{ Propranolol } \\
\hline 2 & $3771.6 \pm 233.4^{b}$ & 37 \\
\hline 3 & $3661.6 \pm 622.3$ & 39 \\
\hline \multicolumn{3}{|c|}{ Propranolol and Pyrimethamine } \\
\hline $2+50$ & $871.0 \pm 486.9^{b}$ & 86 \\
\hline $3+50$ & $144.5 \pm 84.1 \mathrm{~b}$ & 98 \\
\hline
\end{tabular}

Pyrimethamine, Pos. control

50

$3532 \pm 38.8$

41

PBS, Neg. control

$5973.3 \pm 388.8$

$\mathrm{a} \%$ Growth Inhibition $=(1$ - No. of tachyzoites after treatment $/$ No. of tachyzoites in negative control $) \times 100$

$\mathrm{b}$ Indicates a statistically significant difference compared to positive controls $(\mathrm{P}<0.05)$.

Table 2. Anti-Toxoplasma Activity of Propranolol in Mice Peritoneal Exudates (Post-Treatment Group)

\begin{tabular}{|lcc}
\hline Drug/Dose, $\mathbf{m g}$ & Tachyzoites No. $\left(\times \mathbf{1 0}^{\mathbf{4}}\right)$ & \% Growth Inhibition \\
\hline Propranolol & & 75 \\
\hline 2 & $1526.6 \pm 171.4 \mathrm{~b}$ & 51 \\
\hline 3 & $2948.3 \pm 1452.8^{\mathrm{b}}$ & 99.9 \\
\hline Propranolol and Pyrimethamine & & 99.9 \\
\hline $2+50$ & $2 \pm 1^{\mathrm{b}}$ & 99.9 \\
\hline $3+50$ & $6.6 \pm 2.0$ & \\
\hline Pyrimethamine (Pos. control) & & \\
\hline 50 & $8 \pm 2$ & - \\
\hline PBS (Neg. control) & & \\
\hline- & $5990.0 \pm 312.2$ & \\
\hline
\end{tabular}


$\mathrm{a} \%$ Growth Inhibition = (1- No. of tachyzoites after treatment $/$ No. of tachyzoites in negative control $) \times 100$.

$\mathrm{b}$ Indicates a statistically significant difference compared to positive controls $(\mathrm{P}<0.05)$

\section{Discussion}

The concept of host targeting for the prevention and treatment of infectious diseases has recently emerged; however, this concept remains unexplored with regards to parasitic infections. Since T. gondii must invade host nuclear cells to proliferate, this interaction between the parasite and host cells could be a potential site for intervention in the control of Toxoplasma infections $(3,16)$. The present study revealed the effects of propranolol in murine acute infection with a highly virulent RH strain of T. gondii and hinted at the discovery of new potential therapies. In an infection with $1 \times 10^{3}$ tachyzoites, propranolol with doses at 2 and $3 \mathrm{mg} / \mathrm{kg} /$ day in combination with pyrimethamine ( $50 \mathrm{mg} / \mathrm{kg}$ BW/day) was remarkably effective against $T$. gondii in vivo. The growth inhibition rate using propranolol 2 and $3 \mathrm{mg} / \mathrm{kg}$ was $86 \%$ and $98 \%$ in the pre-treated group and $99.97 \%$, and $99.99 \%$ in the posttreated group, respectively. In addition, similar effects were observed in this survey in propranolol at different doses ( 2 and $3 \mathrm{mg} / \mathrm{kg} /$ day) with growth inhibition at 37\% and $39 \%$ compared to pyrimethamine with $41 \%$ growth inhibition in pre-treatment groups.

In the post-treatment group, pyrimethamine with 99.99\% growth inhibition, compared to propranolol at 2 and $3 \mathrm{mg} / \mathrm{kg} /$ day with a growth inhibition of $75 \%$, and $51 \%$, respectively, was highly effective against toxoplasmosis. Of course, propranolol at 2 and $3 \mathrm{mg} / \mathrm{kg} /$ day combined with pyrimethamine resulted in the highest response. Martins-Duarte et al. performed a similar in vivo study and observed that fluconazole combined with sulfadiazine and pyrimethamine was highly effective against $T$. gondii (16). Combination therapy is known as the most effective treatment for toxoplasmic encephalitis $(1,17)$. Pyrimethamine monotherapy for the treatment of toxoplasmosis is not recommended, since patients receiving maintenance treatment with pyrimethamine alone were susceptible to relapses of toxoplasmosis (18).

Furthermore, combined treatment can lead to drug dose reduction and consequently fewer side effects. Our result showed that administration of a combination of drugs might lead to a reduction in drug dosages and injections. This observation is very interesting, since propranolol at a dose of $2 \mathrm{mg} / \mathrm{kg} /$ day in the pre-treatment and posttreatment group had growth inhibition at 37\% and 75\%, respectively. Thus using a combination of new drugs is a promising approach for new anti-Toxoplasma drug discovery. Our findings suggest a synergistic effect of propranolol plus pyrimethamine in vivo. As previously mentioned, a few studies have assessed the efficacy of these drugs in infectious diseases, especially parasitic diseases. Ohnishi et al., investigated the effects of several membrane-acting drugs on malaria and sickle cell anemia and found that propranolol inhibits the growth of Plasmodium falciparum (in vitro) and P. vinckei (in vivo) (19).

In another study, the effect of 22 pharmacological agents on the enhancement of the antimalarial activity of chloroquine was evaluated in a chloroquine-resistant line of Plasmodium yoelii. The study showed that only the cyproheptadine-chloroquine combination produced a curative response; the propranolol-chloroquine combination was inactive (20).

Murphy et al. demonstrated that erythrocyte G proteins are functional, and that propranolol, an antagonist of $G$ protein-coupled b-adrenergic receptors, inhibits $G$ protein activity in erythrocytes. They also discovered that, similar to other b2-antagonists, propranolol inhibited parasite growth during the blood stage. Thus, when propranolol is used in combination with existing antimalarials in cell culture, reduces by $50 \%$ and $90 \%$ inhibitory concentrations for existing drugs against P. falciparum. The combination was also effective in reducing the drug dose in animal models of infection (21). It is well established that propranolol has membrane-stabilizing activity by increasing potassium efflux and decreasing water content $(15,22)$. One obvious possible disadvantage of these drugs is their potential toxicity and side effects. Propranolol is approved for human use and typically prescribed at $0.8-4.5 \mathrm{mg} / \mathrm{kg} /$ day. It is also recommended for use in pregnant women. Thus, it may be an appropriate drug for the treatment of congenital toxoplasmosis. Overall, high concentrations of propranolol can also be administered with few serious side effects (23).

In conclusion, our results elucidated the promising prophylactic and therapeutic effects of propranolol and demonstrated the increased efficiency of the propranolol-pyrimethamine combination against T. gondii in vivo. This novel drug combination in the treatment of toxoplasmosis could lead to fast recovery and few relapses of the disease. There is also an opportunity to decrease the pyrimethamine dosage and its related side effects by using such combinations.

\section{Authors' Contributions}

Ahmad Daryani, Mohammad Ali Ebrahimzadeh, Mehdi Sharif, and Ehsan Ahmadpour designed the research. Shahabeddin Sarvi, Mahbobeh Montazeri, and Ehsan Ahmadpour performed laboratory testing. All authors prepared this manuscript.

\section{Funding/Support}

This study was prepared from M. Montazeri's MSc thesis and supported by a grant (no. 92-471) from the deputy of research, Mazandaran university of medical sciences, Sari, Iran. 


\section{References}

1. Petersen E. Toxoplasmosis. Semin Fetal Neonatal Med. 2007;12(3):214-23.

2. Weiss LM, Dubey JP. Toxoplasmosis: A history of clinical observations. Int J Parasitol. 2009;39(8):895-901.

3. Weiss LM, Kim K. Toxoplasma gondii: the model apicomplexan. Perspectives and methods. Academic Press; 2011.

4. Ambroise-Thomas P, Pelloux H. Toxoplasmosis - congenital and in immunocompromised patients: a parallel. Parasitol Today. 1993;9(2):61-3.

5. Serranti D, Buonsenso D, Valentini P. [Congenital toxoplasmosis treatment]. Eur Rev Med Pharmacol Sci. 2011;15(2):193-8.

6. Al-Zanbagi NA. Effectiveness of Myrrh and Spiramycin as Inhibitors for Toxoplasma gondii Tachyzoites in vivo. Mansoura J Forensic Med Clin Toxicol. 2007;15(2):117-28.

7. Araujo FG, Remington JS. Recent advances in the search for new drugs for treatment of toxoplasmosis. Int J Antimicrob Agents. 1992;1(4):153-64.

8. Ryning FW, Remington JS. Effect of cytochalasin D on Toxoplasma gondii cell entry. Infect Immun. 1978;20(3):739-43.

9. Morisaki JH, Heuser JE, Sibley LD. Invasion of Toxoplasma gondii occurs by active penetration of the host cell. J Cell Sci. 1995;108 ( Pt 6):2457-64.

10. D'Angelo JG, Bordon C, Posner GH, Yolken R, Jones-Brando L. Artemisinin derivatives inhibit Toxoplasma gondii in vitro at multiple steps in the lytic cycle. J Antimicrob Chemother. 2009;63(1):146-50.

11. Daryani A, Ebrahimzadeh MA, Sharif M, Rezaei F, Ahmadpour E, Sarvi S. The inhibitory effect of ketotifen on entrance of Toxoplasma gondii tachyzoites into macrophages of mouse. J Mazandaran Univ Med Sci. 2014;23(110):75-80.

12. Daryani A, Hosseini AZ, Dalimi A. Immune responses against excreted/secreted antigens of Toxoplasma gondii tachyzoites in the murine model. Vet Parasitol. 2003;113(2):123-34.
13. Yang CD, Chang GN, Chao D. Protective immunity against Toxoplasma gondii in mice induced by the SAG2 internal image of anti-idiotype antibody. Parasitol Res. 2003;91(6):452-7.

14. Dadimoghaddam Y, Daryani A, Sharif M, Ahmadpour E, Hossienikhah Z. Tissue tropism and parasite burden of Toxoplasma gondii RH strain in experimentally infected mice. Asian PacJTrop Med. 2014;7(7):521-4.

15. Langslet A. Membrane stabilization and cardiac effects of d,1propranolol, d-propranolol and chlorpromazine. Eur J Pharmacol.1970;13(1):6-14.

16. Martins-Duarte ES, de Souza W, Vommaro RC. Toxoplasma gondii: the effect of fluconazole combined with sulfadiazine and pyrimethamine against acute toxoplasmosis in murine model. Exp Parasitol. 2013;133(3):294-9.

17. Fung HB, Kirschenbaum HL. Treatment regimens for patients with toxoplasmic encephalitis. Clin Ther.1996;18(6):1037-56.

18. Foppa CU, Bini T, Gregis G, Lazzarin A, Esposito R, Moroni M. A retrospective study of primary and maintenance therapy of toxoplasmic encephalitis with oral clindamycin and pyrimethamine. Eur J Clin Microbiol Infect Dis. 1991;10(3):187-9.

19. Ohnishi ST, Sadanaga KK, Katsuoka M, Weidanz WP. Effects of membrane acting-drugs on plasmodium species and sickle cell erythrocytes. Mol Cell Biochem. 1989;91(1-2):159-65.

20. Singh N, Puri SK. Interaction between chloroquine and diverse pharmacological agents in chloroquine resistant Plasmodium yoelii nigeriensis. Acta Trop. 2000;77(2):185-93.

21. Murphy SC, Harrison T, Hamm HE, Lomasney JW, Mohandas N, Haldar K. Erythrocyte $G$ protein as a novel target for malarial chemotherapy. PLoS Med. 2006;3(12):e528.

22. Manninen V. Movements of sodium and potassium ions and their tracers in propranolol-treated red cells and diaphragm muscle. Acta Physiol Scand Suppl. 1970;355:1-76.

23. Porter RS, Kaplan JL. The Merck manual of diagnosis and therapy 18th ed. Rahway, New Jersey: Merck Research Laboratories; 